\title{
Multifocal ectopic thyroid tissues including breast: A case report
}

\author{
AHRIM MOON $^{1}$, HYUN-SOO KIM $^{2}$, KYUNGSEEK CHANG $^{3}$ and SUNG-IM DO ${ }^{3}$ \\ ${ }^{1}$ Department of Pathology, Soonchunhyang University Bucheon Hospital, Soonchunhyang University College of Medicine, \\ Bucheon, Gyeonggi 14584; ${ }^{2}$ Department of Pathology, Severance Hospital, Yonsei University College of Medicine, \\ Seoul 03722; ${ }^{3}$ Department of Pathology, Kangbuk Samsung Hospital, Sungkyunkwan \\ University School of Medicine, Seoul 03181, Republic of Korea
}

Received January 11, 2019; Accepted August 14, 2019

DOI: $10.3892 /$ mco.2019.1968

\begin{abstract}
The current study reports the case of multifocal ectopic thyroid tissues in the breast. Ectopic thyroid tissue is an uncommon entity found not only in the cysts of thyroglossal ducts, but also along the thyroglossal duct. The most frequent location of this tissue is the base of the tongue, but they can also occur in the anterior tongue, submandibular or sublingual region, larynx, trachea, mediastinum and heart. Only a single case of ectopic thyroid tissue in breast has been previously reported in English literature. However, the current case was associated with abnormal embryological development compared with previous reports, which are attributed to abnormal implantation.
\end{abstract}

\section{Introduction}

Ectopic thyroid tissue is a rare condition resulting from abnormal embryological development and migration of thyroid gland. It can be found anywhere along the path of the thyroid descent, from the floor of the primitive foregut to the final position of the thyroid in the neck. The estimated frequency of ectopic thyroid is 1 per 100,000 to 300,000 individuals (1), with lingual thyroid accounting for $90 \%$ of cases (2). Extra-lingual ectopic thyroid is less frequently encountered and its location in breast is extremely rare. According to the literature review performed by the authors this is the first case of multifocal ectopic thyroid tissue including breast. Ectopic intra-mammary thyroid can be confused with other diseases such as cysts, intra-mammary lymph nodes, and fat necrosis.

Correspondence to: Dr Sung-Im Do, Department of Pathology, Kangbuk Samsung Hospital, Sungkyunkwan University School of Medicine, 29 Saemunan-ro, Jongno-gu, Seoul 03181, Republic of Korea

E-mail: sungim.do@samsung.com

Key words: ectopic thyroid tissue

\section{Case report}

A 53-year-old woman was admitted to Kangbuk Samsung Hospital (Seoul, Korea) to confirm the breast lesion. She visited our Breast and Thyroid Center due to multiple nodular lesions in the left breast, which were detected during routine radiological examinations for more than 10 years. She has a surgical history of right thyroidectomy due to nodular hyperplasia diagnosed 16 years ago. In addition, she had excision for palpable masses in the right medial infraclavicular lesion (measuring $1.6 \mathrm{~cm}$ in the largest diameter) and left sternoclavicular junction (1.4 cm in the largest diameter) 10 years ago. The pathologic diagnosis for masses was ectopic thyroid. Thyroid function tests assessing TSH and Free T4 were carried out every year and showed euthyroidism status. Because the patient's thyroid function was normal, the patient did not receive any medications or thyroid hormone supplements, except vitamin D3. Ultrasonography performed 10 years ago showed multiple scattered cysts in bilateral breast. The largest lesion was located in the left breast and measured about $0.5 \mathrm{~cm}$ in size (Fig. 1). They were thought to be benign findings such as cysts, intra-mammary lymph nodes or fat necrosis based on Breast Imaging-Reporting and Data System 2 criteria. No associated inflammatory signs or skin changes were detected.

However, a recent radiological study showed an increase in the size and area of left breast lesion. Radiological breast examination including mammography and ultrasonography revealed multiple, well-defined, oval nodules in the inner and upper area measuring less than $1 \mathrm{~cm}$ and located in the subcutaneous fat layer. The lesions revealed segmental distribution and occasionally increased vascularity, warranting pathologic confirmation. No abnormal findings were detected in both axillae. The patient underwent core needle biopsy of the breast lesion to establish the diagnosis. Five portions of breast tissue, including the largest one measuring $1.5 \times 1.0 \mathrm{~cm}$, were submitted.Microscopic evaluation confirmed the presence of thyroid tissue with predominantly macrofollicular structures, but normal surrounding breast parenchyma. The thyroid tissue tested positive for thyroid transcription factor-1 based on immunohistochemistry staining (Fig. 2). The thyroid function was normalized after the surgery based on TSH levels of $1.49 \mu \mathrm{IU} / \mathrm{ml}$, and free T4 concentrations of $1.18 \mathrm{ng} / \mathrm{dl}$. 


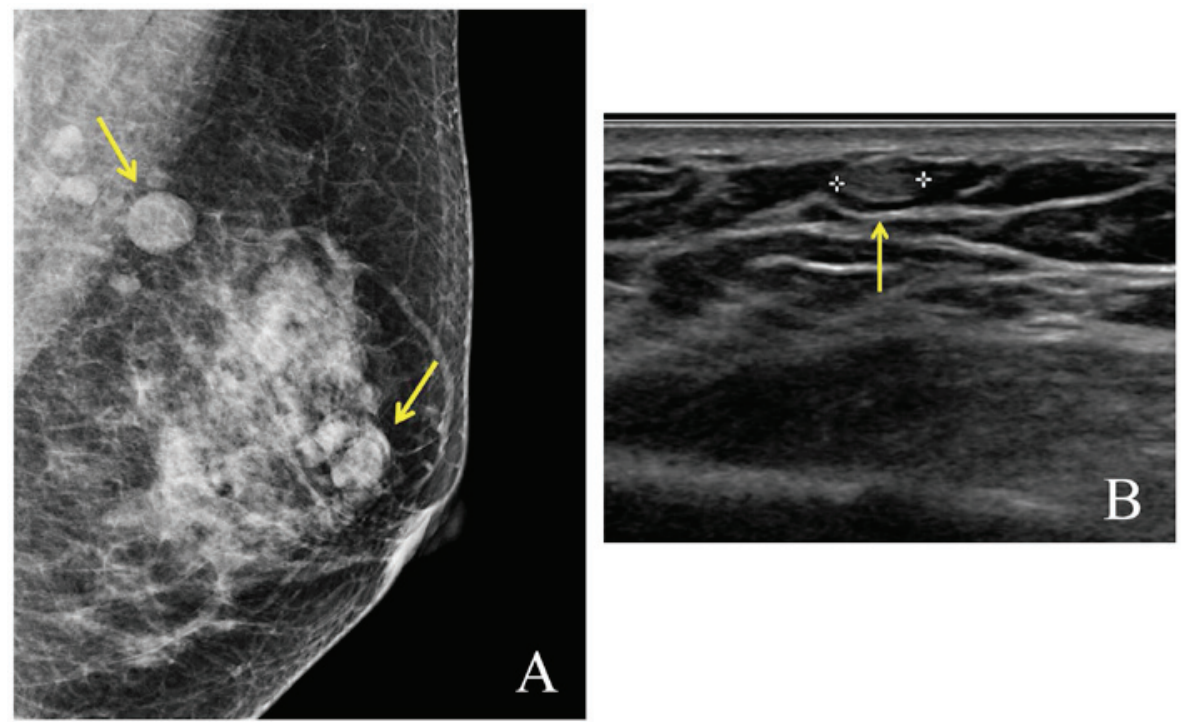

Figure 1. (A) The mammography and (B) ultrasonography of patient shows multiple well demarcated nodules in left breast (arrows). Radiologist reported this lesion as Breast Imaging-Reporting and Data System 2.
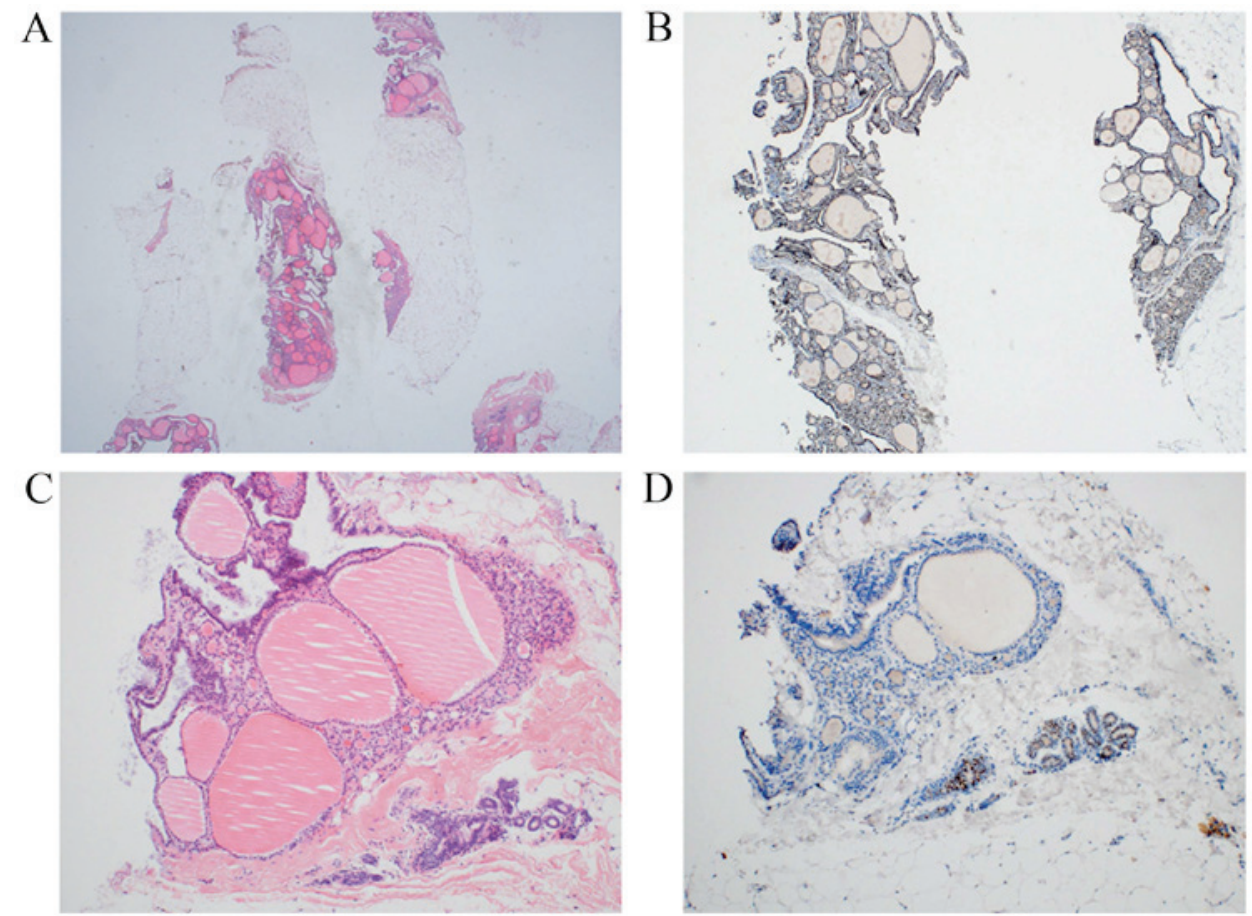

Figure 2. (A) The hematoxylin and eosin stain slide of received core biopsied tissue shows thyroid follicles in fatty tissue. (B) These follicular cells are strongly positive for thyroid transcription factor-1 immunohistochemistry staining and consistent with thyroid follicles. (C) On higher magnification, there are some normal mammary ducts and (D) they indicated estrogen receptor expression.

\section{Discussion}

This is the first reported case of multiple ectopic thyroid in breast. We found a previous report of thyroidal nodule in the right breast of a 19-year-old woman (3). However, the patient had a history of endoscopic right thyroidectomy through breast. Pathology report of the tissue specimen indicated the presence of follicular tissue at the drainage incision site and the inner canal. Therefore, the thyroid nodule in the breast involved grafted thyroid cells at the drainage incision site. Interestingly, our case patient also had a history of previous thyroid lobectomy. However, she underwent right thyroid lobectomy and ectopic thyroid tissue was located in the left breast. Therefore, the possibility of thyroid tissue implant in breast during surgery was very low. It was not possible to compare with similar cases because no other studies reported multiple ectopic thyroid tissues in the breast.

The most common reason for ectopic thyroid tissue involved inhibition of migration or excessive descent of the thyroid anlage. Therefore, it was found along the path of descent in the developing thyroid primordium from the foramen cecum. The lingual area was the most common site in about $90 \%$ of 
all cases, with a majority of the cases associated with the base of tongue (2). Clinical reports of extra-lingual ectopic thyroid tissue are rare. We found a few studies of ectopic thyroid in adrenal gland (4,5), gallbladder (6), jejunum (7) and mesentery (8) but not pure ectopic thyroid in the breast. The etiology of this abnormality is not fully understood. However, genetic factors and mutations in the regulatory genes expressed in the developing thyroid have been implicated in human thyroid ectopy $(2,9)$. Ectopic thyroid tissue in the breast is rare and is difficult to explain based on abnormal migration as it is distant from the path of embryological development.

The treatment of ectopic thyroid tissue depends on factors such as mass size, symptoms, age of the patient, thyroid functional status and histological findings (10). Surgical excision is often the treatment of choice in symptomatic cases, and other options include hormone suppression and radioactive iodine-131 ablation (11) based on the different factors. In this case, iodine-131 scan was not carried out because right thyroidectomy was performed and the opposite thyroid lobe remained. Hypothyroidism occurs in about $33 \%$ of patients with ectopic thyroid tissue and euthyroidism has been reported in $61 \%$ of such patients (9). The difference in thyroid function status may be attributed to heterogeneous age distribution and location of ectopic thyroid tissue. Hypothyroidism was prevalent in the lingual area with a lower mean age and without an eutopic thyroid (9). The majority of patients are generally asymptomatic while a few cases are detected incidentally. Symptoms are usually related to the size and location of the ectopic tissue as well as associated endocrine dysfunction.

It is also important to distinguish metastases from thyroid carcinoma. Distant metastases of differentiated thyroid carcinomas have been reported anywhere. A previous study reported multiple ectopic thyroids in the lung, which might be confused with pulmonary metastasis (12). Multiple ectopic thyroids may be considered in a patient with a history of thyroid carcinoma. However, our patient did not have a cancer history and the thyroid tissue in breast showed normal follicular pattern and cells.

Despite the lack of standard therapy for ectopic thyroid in breast due to its rarity, further surgical treatment was not considered because the patient did not exhibit thyroid hormone-related symptoms. Although ectopic thyroid tissue is benign, it has a tendency to show enlargement and manifest compression symptoms. In addition, the possibility of carcinomatous transformation such as papillary carcinoma arising in struma ovarii should be considered. Therefore, a regular follow-up is important.

According to the literature review, this is the first case of ectopic thyroid tissue in the breast, which underscores the significance of our report. Additional cases and further discussion are needed to develop an appropriate treatment protocol or intervention.

\section{Acknowledgements}

Not applicable.

\section{Funding}

No funding was received.

\section{Availability of data and materials}

The datasets used and/or analyzed during the present study are available from the corresponding author on reasonable request.

\section{Authors' contributions}

$\mathrm{KC}$ is acquired the data, performed the literature review and wrote the manuscript. AM, HSK, SID acquired the data and contributed clinical advice. AM evaluated the images and SID evaluated the specimens. All authors read and approved the final manuscript.

\section{Ethics and consent to participate}

Not applicable.

\section{Patient consent for publication}

Informed consent was obtained from the patient about the publication of the case details and any associated images.

\section{Competing interests}

The authors declare that they have no competing interests.

\section{References}

1. Williams ED, Toyn CE and Harach HR: The ultimobranchial gland and congenital thyroid abnormalities in man. J Pathol 159: 135-141, 1989.

2. Ibrahim NA and Fadeyibi IO: Ectopic thyroid: Etiology, pathology and management. Hormones (Athens) 10: 261-269, 2011.

3. Ye MN, Zhang WH, Yuan YX, Zhang XY and Chen HF: Thyroid nodule of the breast. Breast J 22: 240-243, 2016.

4. Casadei GP, Bertarelli C, Giorgini E, Cremonini N, de Biase D and Tallini G: Ectopic thyroid tissue in the adrenal gland: Report of a case. Int J Surg Pathol 23: 170-175, 2015.

5. Gourmaud J, Bongiovanni M, Triponez F and Pusztaszeri M: Ectopic thyroid tissue in the adrenal gland. Endocr Pathol 25: 353-355, 2014.

6. Cassol CA, Noria D and Asa SL: Ectopic thyroid tissue within the gall bladder: Case report and brief review of the literature. Endocr Pathol 21: 263-265, 2010.

7. Hammers YA, Kelly DR, Muensterer OJ, Hardin WD Jr, Saeed SA and Mroczek-Musulman EC: Giant polypoid gastric heterotopia with ectopic thyroid tissue: Unusual cause of jejuno-jejunal intussusception. J Pediatr Gastroenterol Nutr 45: 484-487, 2007.

8. Güngör B, Kebat T, Ozaslan C and Akilli S: Intra-abdominal ectopic thyroid presenting with hyperthyroidism: Report of a case. Surg Today 32: 148-150, 2002.

9. Zheng W, Tan J and Liu T: Coexistence of non-functional ectopic thyroid tissue and a normal thyroid: A case report. Exp Ther Med 6: 1059-1061, 2013.

10. Guerra G, Cinelli M, Mesolella M, Tafuri D, Rocca A, Amato B, Rengo S and Testa D: Morphological, diagnostic and surgical features of ectopic thyroid gland: A review of literature. Int J Surg 12 (Suppl 1): S3-S11, 2014.

11. Rahalkar M, Rahalkar A and Solav S: A rare case of triple thyroid ectopia. Indian J Endocrinol Metab 18: 238-240, 2014.

12. Ryu HS, Chung YJ, Chong S and Lee JI: Ectopic intrapulmonary thyroid tissue mimicking metastatic tissue. Thyroid 22: 755-759, 2012. 